\title{
An efficient probabilistic approach to vibro-acoustic analysis based on the Gaussian orthogonal ensemble
}

\author{
Edwin Reynders ${ }^{\text {a) }}$ \\ Department of Civil Engineering, KU Leuven, Kasteelpark Arenberg 40, B-3001 Leuven, Belgium \\ Julien Legault and Robin S. Langley \\ Department of Engineering, University of Cambridge, Trumpington Street, Cambridge CB2 1PZ, \\ United Kingdom
}

(Received 3 July 2013; revised 17 April 2014; accepted 18 May 2014)

\begin{abstract}
Vibro-acoustic analysis of complex systems at higher frequencies faces two challenges: how to compute the response without using an excessive number of degrees of freedom (DOFs), and how to quantify the uncertainty of the response due to small spatial variations in geometry, material properties, and boundary conditions, which have a wave scattering effect? In this study, a general method of analysis is presented that provides an answer to both questions while overcoming most limitations of statistical energy analysis. The fundamental idea is to numerically compute an artificial ensemble of realizations for the components of the built-up system that are highly sensitive to small random wave scatterers. This can be efficiently performed because their eigenvalue spacings and mode shapes conform to Gaussian orthogonal ensemble spacings and Gaussian random fields, respectively. The DOFs of the overall system are therefore limited to those of the deterministic components and the interface DOFs of the random components. The method is extensively validated by application to plate structures. Good agreement between the predicted response probability distributions and the results of detailed parametric probabilistic models is obtained, also for cases of low modal overlap, single point loading, and strong subsystem coupling.

(C) 2014 Acoustical Society of America. [http://dx.doi.org/10.1121/1.4881930]
\end{abstract}

PACS number(s): 43.40.Hb, 43.40.Dx, 43.20.Ks, 43.55.Rg [LLT]

Pages: 201-212

\section{INTRODUCTION}

The analysis of the dynamic response of structural and acoustic systems at higher frequencies faces two major challenges. The first one is that of computational efficiency: Given the short wavelengths that appear at high frequencies, how can the response of the structure be computed without using an excessive number of degrees of freedom (DOFs)? The second one is that of robustness: Given that the local response at higher frequencies is very sensitive to wave scattering caused by spatial variations in geometry, material properties, and boundary conditions, ${ }^{1}$ how can the uncertainty of the response, due to the very many uncertain model parameters that affect it, be quantified?

Deterministic element-based methods such as the finite element method (FEM) and the boundary element method (BEM) in their standard form are not well suited for midand high-frequency analysis. This is because they require a very small element size to capture the short-wavelength response with sufficient accuracy ${ }^{2}$ resulting in a high computation cost even for a single deterministic model. Analyzing an ensemble of randomized models further increases the computation cost. ${ }^{3,4}$ At high frequencies, where all parts of a built-up system are very sensitive to such variations, stochastic energy-based methods like statistical energy analysis (SEA) can be employed. In this approach, the system is decomposed into subsystems that are supposedly

\footnotetext{
a) Author to whom correspondence should be addressed. Electronic-mail: edwin.reynders@bwk.kuleuven.be
}

homogenous and weakly coupled. The response of each subsystem is characterized with a single random variable, its total vibrational energy, and the interaction between subsystems is described by means of a power balance. SEA is computationally very efficient because (i) it characterizes the response of each subsystem by means of a global instead of a local response quantity and (ii) it employs a nonparametric model of uncertainty, which implies that the detailed statistics of the underlying physical parameters are not needed for computing the energy statistics. Until recently only the mean energy values could be reliably estimated. ${ }^{5}$ Useful information could therefore be obtained only at high modal overlap, where the probability of the response is narrowly distributed around the mean value. Langley and Cotoni extended the SEA method to predicting the variance of the energy by introducing an additional set of parameters that describe the nature of the external power input to each subsystem and the coupling between the different subsystems. ${ }^{6}$ Very recently the marginal probability density function (PDF) of a subsystem's total energy has been characterized for some special but important cases. Under the additional assumption of moderately high modal overlap and for certain loading cases, the energy is approximately lognormally distributed. ${ }^{7}$ For high modal overlap it is chi-squared distributed. ${ }^{8}$

When some but not all subsystems of a built-up system are very sensitive to small wave scatterers, neither the lowfrequency displacement-based approach nor the highfrequency statistical energy approach is adequate. However, in this mid-frequency range, a hybridization of both has been achieved, based on a reciprocity relationship that links the 
mean vibrational energy of a nonparametric random SEA subsystem to the variance of the nodal forces at its boundary. ${ }^{9}$ Explicit expressions for the mean ${ }^{10}$ and variance ${ }^{11}$ of the response of all system components have been derived. Their marginal probability distributions have also been derived under the additional assumption that the SEA subsystems have high modal overlap. ${ }^{8}$ The hybrid approach has been validated in industrial applications. ${ }^{12}$

The applicability of SEA-based methods is limited however. First, only the marginal probability distribution of the response can be predicted with reasonable accuracy, and only when all random subsystems have sufficiently high modal overlap and when they are loaded in a specific way. Nevertheless, the probability distribution of the response is needed for computing, e.g., the probability that a certain response level is exceeded, or for computing confidence intervals on system response quantities. A second limitation stems from the fundamental assumption that all subsystems are weakly coupled to each other and failure of SEA has been observed for systems that are strongly coupled according to some measure ${ }^{13-16}$ (as noted by Finnveden, ${ }^{17}$ there is no generally agreed definition of coupling strength in this context).

The aim of this paper is to develop a robust mid- and high-frequency analysis method that is computationally cheap when compared to FEM and related approaches but that does not have the limitations of SEA as discussed above. It also employs a nonparametric model of uncertainty for spatial variations in geometry, material properties and boundary conditions. The main difference with SEA-based approaches, however, is that the response of a nonparametric random subsystem is characterized by its full displacement field instead of its total energy. With this approach it is possible to couple a nonparametric random subsystem to any other type of subsystem without making additional assumptions such as weak coupling, and no additional assumptions are needed for computing the probability distribution of the response. Furthermore, compared to SEA, the computation of Green's functions for evaluating the direct field dynamic stiffness matrix, ${ }^{9}$ which represents the behavior of a subsystem in the absence of reflections of incoming waves, is not necessary, and the computational effort does not increase when additional parametric uncertainty (e.g., in the damping parameters) is considered.

The method makes use of the fact that, when a homogenous elastodynamic system is highly sensitive to wave scattering caused by spatial variations in geometry, material properties, and boundary conditions, the statistics of its undamped eigenvalues and mode shapes saturate to universal distributions. This can be viewed as a central limit result, in the sense that the statistics of the modal characteristics can be described without having any detailed knowledge of the nature of the underlying uncertain physical parameters. ${ }^{11}$ Experimental $^{18-20}$ and numerical ${ }^{21,22}$ studies have shown that the statistics of the local spacings between the eigenvalues saturate to those of the Gaussian orthogonal ensemble (GOE) matrix from random matrix theory. ${ }^{23}$ This random matrix was first introduced in nuclear physics to represent a random Hamiltonian ${ }^{24-26}$ and it bears little resemblance to the matrices that arise in the mathematical description of vibro-acoustic systems. This suggests a kind of universality in the local eigenvalue spacing statistics of the GOE, in the sense that they are valid for a much wider class of systems than can be described by a GOE matrix; ${ }^{27}$ they have indeed already found successful applications in vibro-acoustics, e.g., in the development of the SEA variance theory. ${ }^{6,11}$ As for the mode shapes, these arise as the sum of many random traveling wave components. They therefore saturate to Gaussian random fields, also termed reverberant or wave chaos fields. Their covariance function depends only on the distance between the considered mode shape components and the wavelength. ${ }^{28,29}$ These results will be employed here for the analysis of built-up systems.

The fundamental idea is to numerically compute an artificial ensemble of realizations of the system, where the modal characteristics of the short-wavelength random subsystems are directly generated from the GOE and normal probability distributions. All joint response statistics are straightforwardly obtained from this Monte Carlo ensemble. The same statistics would be obtained by computationally expensive, detailed modeling of all subsystems with e.g., FEM and introducing random parameters to describe spatial variations in geometry, material properties, and boundary conditions.

It should be noted that the GOE-based nonparametric uncertainty approach that is developed here is fundamentally different from the nonparametric uncertainty approach that has been previously introduced by Soize. ${ }^{30-32}$ This approach concentrates on the low- and mid-frequency ranges. It accounts for uncertainties caused by modeling errors, by replacing the deterministic operators (such as mass, stiffness, and damping operators) of the nominal model by random operators and by subsequently constructing sets of random matrices corresponding to these operators (such as random mass, stiffness, and damping matrices) using the maximum entropy principle. In contrast, the present work is concerned with the mid- and high-frequency ranges and with accounting for uncertainties caused by small random wave scatterers.

In what follows, the analysis of a single subsystem is discussed in further detail in Sec. II. The step toward builtup systems is made in Sec. III. In Sec. IV, the method is applied to plate structures, and the computed response statistics are compared with the results of a detailed parametric uncertainty model. The examples also illustrate the applicability of the method to structures that cannot be accurately analyzed with SEA. Conclusions are drawn in Sec. V.

\section{RANDOM SUBSYSTEM MODEL}

The response of a decoupled subsystem displaying highfrequency behavior is analyzed in this section. The subsystem is taken to be homogenous in the sense that it exhibits only global modes, i.e., modes that are not localized in part of the subsystem. A nonparametric uncertainty model that accounts for random wave scattering is introduced.

\section{A. Frequency response function}

The following analysis is concerned with the vibration response of a decoupled subsystem that has a scalar response 
variable $u_{k}(\mathbf{x}, t)$ representing the displacement of the subsystem at spatial location $\mathbf{x}$ and time $t$. The subsystem number is denoted by subscript $k$. The subsystem is taken to have proportional damping, with loss factor $\eta_{k}$. A distributed harmonic load of frequency $\omega$ is applied. It is characterized by a complex amplitude function $f_{k}(\mathbf{x}, \omega) \in \mathbb{C}$ so that its time history is obtained as $f_{k}(\mathbf{x}, t)=\operatorname{Re}\left[f_{k}(\mathbf{x}, \omega) e^{i \omega t}\right]$. Similarly, the subsystem response is characterized by a displacement amplitude function $u_{k}(\mathbf{x}, \omega) \in \mathbb{C}$. It can be obtained from

$$
u_{k}(\mathbf{y}, \omega)=\int_{\Omega_{k}} H_{k}(\mathbf{x}, \mathbf{y}, \omega) f_{k}(\mathbf{x}, \omega) d \mathbf{x}
$$

where $\Omega_{k}$ denotes the subsystem volume and $H_{k}(\mathbf{x}, \mathbf{y}, \omega)$ its frequency response function (FRF). When written as a modal sum, the FRF reads

$$
H_{k}(\mathbf{x}, \mathbf{y}, \omega)=\sum_{r} \frac{\varphi_{k, r}(\mathbf{y}) \varphi_{k, r}(\mathbf{x})}{-\omega^{2}+i \eta_{k} \omega \omega_{k, r}+\omega_{k, r}^{2}},
$$

where $\omega_{k, r}$ is the $r$ th undamped natural frequency of the decoupled subsystem $k$, and $\varphi_{k, r}(\mathbf{x})$ is the corresponding mode shape. The mode shapes are mass-normalized, i.e.,

$$
\int_{\Omega_{k}} \rho(\mathbf{x}) \varphi_{k, r}^{2}(\mathbf{x}) d \mathbf{x}=1
$$

where $\rho(\mathbf{x})$ denotes the nominal (i.e., mean) mass density.

The natural frequencies of the nonparametric random subsystem are computed via the normalized eigenvalue spacings. The $r$ th normalized spacing for subsystem $k$ is defined as

$$
s_{k, r}:=n_{k}^{\lambda}(\lambda)\left(\lambda_{k, r}-\lambda\right)=\frac{n_{k}(\omega)}{2 \omega}\left(\omega_{k, r}^{2}-\omega^{2}\right),
$$

where $\lambda:=\omega^{2}, \lambda_{k, r}:=\omega_{k, r}^{2}, n_{k}^{\lambda}(\lambda)$ represents the local eigenvalue density and $n_{k}(\omega)$ the modal density. The modal density is the average number of modes per radial frequency across the random ensemble. It can be obtained as the derivative of the smooth (i.e., non-oscillatory) part of the mode count function of the nominal subsystem. ${ }^{5,33}$ When the excitation frequency $\omega$ and (realizations of) the normalized eigenvalue spacings are known, the natural frequencies can be obtained from

$$
\omega_{k, r}=\sqrt{\frac{s_{k, r} 2 \omega}{n_{k}(\omega)}+\omega^{2}} .
$$

Expression (2) for the FRF contains two random quantities for each mode $r$ : the natural frequency $\omega_{k, r}$ or equivalently the normalized spacing $s_{k, r}$, and the mode shape $\varphi_{k, r}(\mathbf{x})$. A nonparametric uncertainty model for these random quantities will be developed in the next sections, where it is assumed that the spacings and the mode shapes are statistically independent from each other. With this assumption, the probability distribution of the response can be computed when the joint probability distribution of the spacings and the joint probability distribution of the mode shapes are known. These distributions are considered in the next two sections.

\section{B. Spacing statistics and the GOE}

This section deals with the efficient computation of the joint probability distribution of the eigenvalue spacings $s_{k, r}$ of a subsystem, representing the uncertainty due to spatial variations in geometry, material properties, or boundary conditions. At first instance, one might think that no general statements about the distribution of the eigenvalue spacings $s_{k, r}$ can be made without knowledge of the detailed distribution of the random parameters underlying their uncertainty. For example, when point masses are randomly attached to a plate, one can expect that the probability distribution of the eigenvalue spacings depends on the total number of point masses, their individual mass, and the joint probability distribution of the locations of attachment. This is true as long as the natural frequencies of the subsystem do not mix well across the random ensemble, i.e., when the random variations of a natural frequency are small compared to the mean spacing between neighboring natural frequencies.

However, it has been shown that, when a subsystem is sufficiently random, then the statistics of the eigenvalue spacings saturate to universal distributions, which are independent from the statistics of the underlying random parameters. ${ }^{18-22}$ This result allows for a nonparametric description of the spacings distribution, i.e., a description that does not depend on the underlying random parameters. The degree of randomness can be quantified by the statistical overlap factor, which is defined as ${ }^{34}$

$$
S_{k}(\omega):=\frac{2 \operatorname{std}\left[\omega_{k, r}\right]}{\mu_{k}(\omega)}=2 n_{k}(\omega) \sqrt{\mathrm{E}\left[\left(\omega_{k, r}-\mathrm{E}\left[\omega_{k, r}\right]\right)^{2}\right]}
$$

where the natural frequency $\omega_{k, r}$ is chosen such that its nominal value $\mathrm{E}\left[\omega_{k, r}\right]$ is closest to the frequency of interest $\omega$. The statistics of the subsystem's eigenvalue spacings tend to saturate to universal distributions when $S_{k}(\omega) \geq 1$. For all types of subsystem, the statistical overlap is monotonically increasing with frequency, so this always happens from a certain frequency onward. The corresponding frequency range is termed the "high-frequency range" of the subsystem.

For the generic case where the considered spatial uncertainty does not preserve subsystem symmetries, the statistics of the local spacings between the eigenvalues saturate to those of the GOE matrix from random matrix theory ${ }^{23}$ at high statistical overlap. In this case the veering (or eigenvalue repulsion) phenomenon prevents the occurrence of double modes, and the probability density function is zero for zero spacing. If on the contrary the nominal subsystem has symmetries that are preserved by the spatial uncertainty, then the eigenvalue spacings follow a Poisson distribution, which allows for multiple modes having the same natural frequency. A subsystem with Poisson natural frequency spacings is mathematically simpler, for instance because the spacings are statistically independent, and historically this case was analyzed first. ${ }^{35}$ However, in general, such symmetry-preserving perturbations are unlikely to occur, and in practical situations GOE behavior is observed. 
The GOE is a real symmetric matrix with random entries that can be written as ${ }^{36}$

$$
\mathbf{G}_{n_{\mathrm{G}}}\left(\sigma_{\mathrm{G}}\right):=\left[\begin{array}{cccc}
G_{11} & G_{12} & \cdots & G_{1 n_{\mathrm{G}}} \\
G_{12} & G_{22} & \cdots & G_{2 n_{\mathrm{G}}} \\
\vdots & \vdots & \ddots & \vdots \\
G_{1 n_{\mathrm{G}}} & G_{2 n_{\mathrm{G}}} & \cdots & G_{n_{\mathrm{G}} n_{\mathrm{G}}}
\end{array}\right],
$$

where $n_{\mathrm{G}}$ denotes the number of rows or columns. The elements with different indices are independent, centered Gaussian random variables. The diagonal elements have variance $2 \sigma_{\mathrm{G}}^{2}$ and the off-diagonal elements have variance $\sigma_{\mathrm{G}}^{2}$, where the parameter $\sigma_{\mathrm{G}}$ serves to specify an eigenvalue scale. It can be noted that the GOE bears little resemblance to the matrices that arise in the mathematical description of vibro-acoustic systems. It is even not positive definite almost surely ${ }^{37}$ and not suitable for the direct description of random mass and stiffness matrices. However, the matrix itself is not of interest here, but rather the joint probability distribution of its normalized eigenvalue spacings, since this is known to be the same distribution as that of the normalized eigenvalue spacings of any vibro-acoustic subsystem in high-frequency regime. This universality of the normalized eigenvalue spacing statistics of the GOE can now be exploited for computing realizations of the spacings $s_{k, r}$.

Let us first approximate the sum in Eq. (2) by retaining only the $N_{k}$ modes for which the normalized eigenvalue distance $\left|s_{k, r}\right|$ is smallest. A realization of the $N_{k}$ normalized spacings $\left\{s_{k, r}\right\}$ can then be computed as follows.

1. Construct a realization of the matrix $\mathbf{G}_{n_{\mathrm{G}}}(\sigma)$, which has been defined in Eq. (7). A Gaussian random number generator can be used for constructing realizations of the elements. The scale factor $\sigma_{\mathrm{G}}$ can be freely chosen as long as it is a finite nonzero positive real number. The number of rows or columns $n_{\mathrm{G}}$ should be chosen such that $n_{\mathrm{G}} \gg N_{k}$.

2. Compute the eigenvalues.

3. Compute and order the spacings around $\lambda=0$. This is a fixed point that is chosen arbitrarily except that it is the center of the eigenvalue range.

4. Normalize the ordered spacings. This can, for instance, be performed by multiplying the spacings by the empirical eigenvalue density at $\lambda=0$.

5. Retain the $n_{r}$ eigenvalue spacings with the smallest absolute value.

An important advantage of this procedure is that the normalized GOE eigenvalue spacings do not depend on any property of the considered subsystem. This implies that the realizations only need to be computed once, thereafter they can be recycled over and over again for analyzing different vibro-acoustic systems. It should be kept in mind though that, when within a single analysis statistically independent subsystems are connected, the corresponding spacing realizations should also be independent. After the spacings have been computed, the corresponding natural frequency realizations of the physical subsystem at hand are obtained from Eq. (5).

Numerical sampling of GOE spacing statistics has been used previously for estimating SEA coupling loss factors, but neglecting the statistical dependency between the spacings. ${ }^{38}$ When neglecting these correlations, the spacing statistics are Rayleigh distributed and realizations of them can be computed directly with a random number generator. However, neglecting these correlations can lead to considerable errors in the response statistics. ${ }^{22}$ Furthermore, in the present paper, the restricting assumptions related to SEA are not made, and the computation of coupling loss factors is not necessary.

\section{Mode shapes as Gaussian random fields}

The mode shapes can be interpreted as standing waves that arise from many diffuse traveling wave components. By virtue of the central limit theorem one can state that, at high frequencies, the mode shapes of the random subsystem are Gaussian random fields. Since the mode shapes are orthogonal, they are also independent from each other. A mode shape component at a single location $\mathbf{x}$ is a zero-mean Gaussian random variable. Its variance can be obtained by taking the expected value of the mass-normalization expression (3):

$$
\int_{\Omega_{k}} \rho(\mathbf{x}) \mathrm{E}\left[\varphi_{k, r}^{2}(\mathbf{x})\right] d \mathbf{x}=1 .
$$

For homogenous subsystems, the random field is stationary, i.e., the averages are independent of the position. In this case we have

$$
\mathrm{E}\left[\varphi_{k, r}^{2}(\mathbf{x})\right]=\frac{1}{\int_{\Omega_{k}} \rho(\mathbf{x}) d \mathbf{x}}=\frac{1}{M_{k}},
$$

where $M_{k}$ is the total mass of subsystem $k$. The modal displacements at points that are far away from each other in terms of wavelength are approximately uncorrelated, and subsystems that are loaded at distinct points can be accurately analyzed under this approximation. ${ }^{39}$

However in other cases the full covariance function of the modal displacement field,

$$
\mathrm{C}_{k, r}(\mathbf{x}, \mathbf{y}):=\mathrm{E}\left[\varphi_{k, r}(\mathbf{x}) \varphi_{k, r}(\mathbf{y})\right]
$$

will be needed. Based on the above arguments, it can be shown that this covariance function is prescribed by ${ }^{28,29}$

$$
\mathrm{C}_{k, r}(\mathbf{x}, \mathbf{y})= \begin{cases}\frac{\mathrm{J}_{0}\left(k_{k, r}\|\mathbf{y}-\mathbf{x}\|\right)}{M_{k}} & \text { for 2D subsystems } \\ \frac{\operatorname{sinc}\left(k_{k, r}\|\mathbf{y}-\mathbf{x}\|\right)}{M_{k}} & \text { for 3D subsystems, }\end{cases}
$$

where $\mathrm{J}_{0}$ denotes the Bessel function of the first kind and order zero, $\operatorname{sinc}(x):=\sin (x) / x$ and $k_{k, r}:=2 \pi / \lambda_{k, r}$ denotes the wavenumber corresponding to the wavelength $\lambda_{r}$ of mode $r$ of subsystem $k$. The covariance depends only on the distance between the mode shape components, the wavelength, and the total mass. Since the wavelength is short compared to the size of the subsystem at high frequencies, 
$k_{k, r}$ can, for instance, be estimated from the deformation of an infinite subsystem at frequency $\omega_{k, r}$.

If necessary, the effect of a deterministic boundary on the mode shape realizations can be accounted for. One possibility is to introduce restrictive boundary conditions (e.g., simply supported) by constraint equations, e.g., by taking the boundary DOFs as part of the master system when solving for the response, as detailed further on in Sec. III B. Boundary reflections can also be accounted for by extending the covariance function by means of an image source approach. ${ }^{40}$

Since a zero-mean Gaussian random field is completely determined by its covariance function, realizations of the mode shapes can be constructed based on the information contained in Eqs. (9) and (11). Realizations for the complete random field can for instance be obtained through a truncated Karhunen-Loève decomposition. ${ }^{3}$ In this approach, the covariance function is decomposed as

$$
\mathrm{C}_{k, r}(\mathbf{x}, \mathbf{y})=\sum_{l} \lambda_{l} f_{l}(\mathbf{x}) f_{l}(\mathbf{y})
$$

where $\lambda_{l}$ and $f_{l}(\mathbf{x})$ are the eigenvalues and eigenfunctions, respectively, of the following eigenvalue problem:

$$
\int_{\Omega_{k}} \mathrm{C}_{k, r}(\mathbf{x}, \mathbf{y}) f(\mathbf{y}) d \mathbf{y}=\lambda f(\mathbf{x}) .
$$

The corresponding mode shape can be expressed as

$$
\varphi_{k, r}(\mathbf{x})=\sum_{l} \sqrt{\lambda_{l}} f_{l}(\mathbf{x}) \xi_{l}
$$

where the $\xi_{l}$ denotes independent, standard normal random variables that can be realized with a Gaussian random number generator. Note that for the covariance functions in Eq. (11), no analytic solution of the eigenvalue problem of Eq. (13) is available at present. A numerical solution is therefore required and this is computationally expensive. ${ }^{41}$ However, when the mode shapes are only needed at a limited number $L_{k}$ of loading locations, the corresponding modal displacements can be collected in a mode shape vector:

$$
\boldsymbol{\varphi}_{k, r}:=\left[\begin{array}{llll}
\varphi_{k, r}\left(\mathbf{x}_{1}\right) & \varphi_{k, r}\left(\mathbf{x}_{2}\right) & \cdots & \varphi_{k, r}\left(\mathbf{x}_{L_{k}}\right)
\end{array}\right]^{T} .
$$

The corresponding covariance matrix is

$$
\mathbf{C}_{k, r}:=\mathrm{E}\left[\boldsymbol{\varphi}_{k, r} \boldsymbol{\varphi}_{k, r}^{T}\right]
$$

It can be straightforwardly computed from the prescribed covariance function Eq. (11). Realizations of the mode shape vector can be obtained from a discrete Karhunen-Loève decomposition: with the eigenvalue decomposition of the covariance matrix

$$
\mathbf{C}_{k, r}:=\mathbf{A} \boldsymbol{\Sigma} \mathbf{A}^{T}
$$

the corresponding mode shape vector is

$$
\boldsymbol{\varphi}_{k, r}:=\mathbf{A} \boldsymbol{\Sigma}^{1 / 2} \boldsymbol{\xi}
$$

where $\xi$ is a vector of independent, standard normal random variables that can be realized with a Gaussian random number generator.

\section{Evaluation of subsystem response}

After a realization of the eigenvalue spacings and the mode shapes has been constructed as detailed in Secs. II B and II C, respectively, the corresponding displacement field can be computed by combining Eqs. (1) and (2):

$$
u_{k}(\mathbf{x}, \omega)=\sum_{r} \frac{\varphi_{k, r}(\mathbf{x}) f_{k, r}}{-\omega^{2}+i \eta_{k} \omega \omega_{k, r}+\omega_{k, r}^{2}},
$$

where $f_{k, r}$ is the modal force for mode $r$ :

$$
f_{k, r}:=\int_{\Gamma_{k}} \varphi_{k, r}(\mathbf{x}) f_{k}(\mathbf{x}, \omega) d \mathbf{x}
$$

If the force is a point load applied at location $\mathbf{x}_{F}$, i.e., when $f_{k}(\mathbf{x}, \omega)=F(\omega) \delta\left(\mathbf{x}-\mathbf{x}_{F}\right)$ with $\delta$ denoting a Dirac impulse, the modal forces are weighted versions of the applied load, where the weights follow from the mode shape realizations:

$$
f_{k, r}:=\varphi_{k, r}\left(\mathbf{x}_{F}\right) F(\omega) .
$$

In the more general case where $f_{k}(\mathbf{x}, \omega)$ is spatially distributed, the modal force $f_{k, r}$ can, from Eq. (20), be interpreted as a weighted sum of correlated, zero-mean normally distributed random variables, the random variables being the mode shapes evaluated at a fixed location and the weights being the loads applied at those locations. Furthermore, when $f_{k}(\mathbf{x}, \omega)$ is random, it is often reasonable to expect that $f_{k}(\mathbf{x}, \omega)$ depends only weakly on any individual mode shape $\varphi_{k, r}(\mathbf{x})$. In this case, $f_{k, r}$ will still have zero mean. When additionally the correlation length of $\varphi_{k, r}(\mathbf{x}) f_{k}(\mathbf{x}, \omega)$ is small compared to the $\Gamma_{k}$, the modal force $f_{k, r}$ can be considered as a sum of many independent, zero-mean random variables, which will be approximately complex normally distributed. ${ }^{42}$ Therefore to simulate realizations of the modal force $f_{k, r}$, only its variance is required. This variance is given by

$$
\mathrm{E}\left[\left|f_{k, r}\right|^{2}\right]=\int_{\Omega_{k}} \int_{\Omega_{k}} \mathrm{E}\left[\varphi_{k, r}(\mathbf{x}) \varphi_{k, r}(\mathbf{y})\right] \mathrm{E}[f(\mathbf{x}) \overline{f(\mathbf{y})}] d \mathbf{x} d \mathbf{y},
$$

where the overbar denotes complex conjugate. For known loading statistics, the integral can be evaluated before computing realizations of the system response. Sometimes the spatial distribution of the loading is known but not the amplitude, e.g., when the loading is described in terms of interface modes with other subsystems. Then the integral can also be evaluated beforehand up to a scaling factor that equals the square of the amplitude.

Beside the local response, the time-averaged total, kinetic and potential energies of a subsystem are also often of interest. With realizations of the natural frequencies and modal forces at hand, they follow from

$$
E_{k}(\omega):=T_{\text {kin }, k}(\omega)+T_{\text {pot }, k}(\omega),
$$




$$
\begin{aligned}
& T_{\text {kin }, k}(\omega)=\frac{\omega^{2}}{4} \sum_{r} \frac{\left|f_{k, r}\right|^{2}}{\left(\omega_{k, r}^{2}-\omega^{2}\right)^{2}+\left(\eta_{k} \omega \omega_{k, r}\right)^{2}}, \\
& T_{\text {pot }, k}(\omega)=\frac{1}{4} \sum_{r} \frac{\omega_{k, r}^{2}\left|f_{k, r}\right|^{2}}{\left(\omega_{k, r}^{2}-\omega^{2}\right)^{2}+\left(\eta_{k} \omega \omega_{k, r}\right)^{2}} .
\end{aligned}
$$

\section{BUILT-UP SYSTEMS}

In this section the step is made from the analysis of a single homogenous random subsystem to the analysis of built-up systems. In order to couple a random subsystem to other random subsystems or even deterministic subsystems, the master system approach ${ }^{10}$ is adopted and extended to include subsystem descriptions in modal form.

\section{A. Stiffness matrix of a random subsystem in modal form}

The response of a random subsystem can be written in modal form as

$$
u_{k}(\mathbf{x}, \omega)=\sum_{r} \varphi_{k, r}(\mathbf{x}) q_{k, r}(\omega),
$$

where the modal coordinates follow from

$$
\left(-\omega^{2}+i \eta_{k} \omega \omega_{k, r}+\omega_{k, r}^{2}\right) q_{k, r}(\omega)=f_{k, r} .
$$

This set of decoupled equations reads in matrix form:

$$
\mathbf{D}_{k}^{\mathrm{m}}(\omega) \mathbf{q}_{k}(\omega)=\mathbf{f}_{k}^{m}(\omega),
$$

where the diagonal matrix $\mathbf{D}_{k}^{\mathrm{m}}(\omega) \in \mathbb{C}^{n_{r} \times n_{r}}$ is the dynamic stiffness matrix of subsystem $k$ in modal form, and the superscript $\mathrm{m}$ denotes that the stiffness and force are in modal coordinates. Realizations of $\mathbf{D}_{k}^{\mathrm{m}}$ are constructed based on GOE natural frequency realizations and Gaussian mode shape realizations. The computation of these has been treated in Sec. II.

\section{B. The master system approach}

In the master system approach, it is assumed that the deterministic subsystems and the subsystem-subsystem and subsystem-force interfaces have been discretized into nodal DOFs. These nodal response DOFs, called master system DOFs, are collected in a displacement amplitude vector $\mathbf{u}(\omega) \in \mathbb{C}^{n_{\mathrm{m}}}$. The equations of motion of the built-up system are then

$$
\mathbf{D}(\omega) \mathbf{u}(\omega)=\mathbf{f}(\omega) .
$$

The dynamic stiffness matrix $\mathbf{D}$ may be decomposed as the sum of the dynamic stiffness matrix of the master system, denoted as $\mathbf{D}_{\mathrm{d}}$, and the dynamic stiffness matrices $\mathbf{D}_{k}$ of the nonparametric random subsystems:

$$
\mathbf{D}(\omega)=\mathbf{D}_{\mathrm{d}}(\omega)+\sum_{k} \mathbf{D}_{k}(\omega) .
$$

For a specific nonparametric random subsystem $k$, let $\boldsymbol{S}_{k} \in \mathbb{R}^{n_{\mathrm{m}, k} \times n_{\mathrm{m}}}$ note a selection matrix collecting the $n_{\mathrm{m}, k}$
DOFs attached to subsystem $k$ from the $n_{\mathrm{m}}$ master system DOFs, and let $\Phi_{k}$ contain the mode shape components at these DOFs:

$$
\boldsymbol{\Phi}_{k}:=\left[\begin{array}{ccc}
\varphi_{k, 1}\left(\mathbf{x}_{1}\right) & \cdots & \varphi_{k, N_{k}}\left(\mathbf{x}_{1}\right) \\
\vdots & \ddots & \vdots \\
\varphi_{k, 1}\left(\mathbf{x}_{n_{\mathrm{m}, k}}\right) & \cdots & \varphi_{k, N_{k}}\left(\mathbf{x}_{n_{\mathrm{m}, k}}\right)
\end{array}\right] .
$$

When $n_{\mathrm{m}, k} \leq N_{k}$, the dynamic stiffness matrix of random subsystem $k$ follows from

$$
\mathbf{D}_{k}(\omega)=\boldsymbol{S}_{k}^{\mathrm{T}}\left(\boldsymbol{\Phi}_{k} \mathbf{D}_{k}^{\mathrm{m}-1}(\omega) \boldsymbol{\Phi}_{k}^{\mathrm{T}}\right)^{-1} \boldsymbol{S}_{k} .
$$

If a realization of the master system response has been constructed, the loading on subsystem $k$ is obtained from

$$
\mathbf{D}_{k}(\omega) \mathbf{u}(\omega)=\mathbf{f}_{k}(\omega) .
$$

The master system approach can be extended rather straightforwardly by allowing $\mathbf{u}(\omega)$ to contain generalized displacements, e.g., modal coordinates of the master system, or coordinates of interface modes between subsystems that are connected by line or area junctions. Such an extension is used further on in the examples of Sec. IV D, where the master system DOFs are the modal coordinates of the master system.

\section{Algorithmic summary}

\section{System definition}

1. Partition the system into a set of coupled subsystems and specify the subsystem interfaces.

2. For each subsystem, estimate the statistical overlap due to uncertainty in spatial variations in geometry, material properties and boundary conditions for the frequency range interest. Subsystems with large statistical overlap are modeled as random subsystems, subsystems with small statistical overlap as deterministic subsystems.

3. Define the master system. It consists of the DOFs of the deterministic subsystems, the subsystem-subsystem interfaces and the subsystem-force interfaces.

\section{Assembly of subsystem dynamic stiffness matrices for each Monte Carlo run and for each random subsystem $k$}

4. Compute a realization of the dynamic stiffness matrix in modal form $\mathbf{D}_{k}^{\mathrm{m}}(\omega)$ based on the realization of a set of GOE spacings as detailed in Sec. II B.

5. Compute a realization of the (generalized) mode shape component matrix Eq. (31) based on the realization of a set of Gaussian mode shapes as detailed in Sec. II C.

6. Compute a realization of the subsystem's dynamic stiffness matrix $\mathbf{D}_{k}(\omega)$ from Eq. (32).

\section{Solution for the system response for each Monte Carlo run}

7. Assemble a realization of the master system's dynamic stiffness matrix as in Eq. (30). 
8. If necessary, introduce additional parametric uncertainty by replacing nominal parameters by a Monte Carlo realization.

9. Construct the (generalized) load vector $\mathbf{f}(\omega)$. If necessary, compute a realization of the generalized forces acting on the subsystems as in Sec. II D.

10. Compute a realization of the master system's response from Eq. (29).

11. Additional quantities may be computed such as a realization of the kinetic energy of a nonparametric random subsystem from Eqs. (24) and (33).

Finally, the solution statistics of interest are computed from the set of Monte Carlo realizations.

\section{APPLICATIONS}

In this section, the proposed method is validated for structures consisting of thin plates connected to stiff components. This type of built-up structure is often encountered in practice, e.g., in an aircraft fuselage where the stiff frames have thin skin panels attached. In the mid-frequency range, the stiff frames exhibit long-wavelength deformation and they are modeled as deterministic, while the thin panels show short-wavelength deformation, and they are modeled as nonparametric random subsystems. The example systems considered here are simple assemblies that capture the physics of this type of problem. The material properties of the plates are the same in all cases: a density of $\rho=2800 \mathrm{~kg} / \mathrm{m}^{3}$, a Young's modulus of $E=7.2 \times 10^{10} \mathrm{~N} / \mathrm{m}^{2}$ and a Poisson's ratio of $\nu=0.3$. In each simulation a total of 5000 independent nonparametric random realizations are computed. For all random subsystems the 50 smallest normalized eigenvalue spacings $s_{k, r}$ are retained. Their realizations are computed with a GOE matrix of dimensions $300 \times 300$. The results are stored and re-used in all examples. The considered frequency range is $100-300 \mathrm{~Hz}$.

The nonparametric model results are compared with "exact" solutions, obtained by a parametric random model constructed with the assumed-modes method, also known as the Lagrange-Rayleigh-Ritz method. ${ }^{43}$ All modes of the detached simply supported plates up to $600 \mathrm{~Hz}$ are taken as Ritz basis functions. The uncertainty that is considered is that of a random mass distribution, in the sense that 20 small masses are attached to the flexible plates at random locations. Each attached mass has $0.75 \%$ of the total mass of the plate. The location of the attached point masses is varied randomly in a total of 1000 Monte Carlo realizations. The parametric stochastic models are computationally expensive compared to the nonparametric models. Figure 1 shows a realization of each of the four investigated systems.

\section{A. Single thin plate, point and line loading}

The first example is that of a thin, simply supported plate of dimensions $2.1 \mathrm{~m} \times 1.9 \mathrm{~m} \times 1.25 \times 10^{-3} \mathrm{~m}$. A particular realization of the plate with random masses attached is shown in Fig. 1(a). Its statistical overlap varies between $3.9($ at $100 \mathrm{~Hz}$ ) and 11.7 (at $300 \mathrm{~Hz}$ ), so the conditions of the GOE model to be valid are fulfilled. (a)

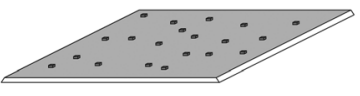

(b)

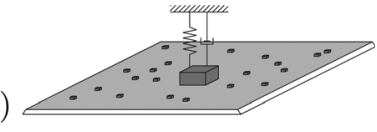

(c)

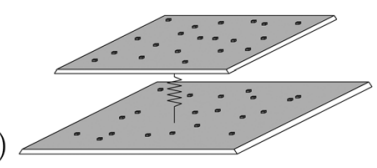

(d)

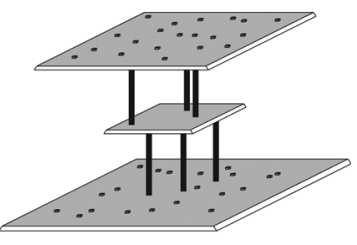

FIG. 1. A single member of an ensemble of structures consisting of (a) a single random plate, (b) an oscillator coupled to a random plate, (c) two random plates, connected by a spring and (d) a deterministic master plate rigidly connected to two random plates at three locations each.

Two load cases are considered: (i) a harmonic point force with amplitude $1 \mathrm{~N}$ at coordinates $(0.88 \mathrm{~m}, 0.72 \mathrm{~m})$ measured along the long and short edges of the plate, respectively, and (ii) a harmonic line load with amplitude $0.78 \mathrm{~N} / \mathrm{m}$ between the points $(0.34 \mathrm{~m}, 0.61 \mathrm{~m})$ and $(1.62 \mathrm{~m}, 0.61 \mathrm{~m})$. The output quantity of interest is the total subsystem energy $E_{1}$. Two different values for the damping loss factor are considered: low damping $(\eta=0.001)$ and normal damping $(\eta=0.01)$.

In both loading cases, the modal forces are Gaussian random variables. For the point load, the variance of the modal force is directly obtained from Eqs. (9) and (21). For the line load, Eqs. (9) and (22) are used. Since the line load is real and constant, and since the covariance function of the mode shapes is known from Eq. (11), Eq. (22) boils down to

$$
\mathrm{E}\left[f_{k, r}^{2}\right]=f_{k}^{2} \int_{0}^{L} \int_{0}^{L} \frac{\mathbf{J}_{0}\left(k_{r}\left|s_{2}-s_{1}\right|\right)}{M_{k}} d s_{1} d s_{2}
$$

where $f_{k}$ denotes the amplitude of the line load $(0.78 \mathrm{~N} / \mathrm{m}$ in this example) and $L$ denotes the length of the line where the load is applied (1.28 $\mathrm{m}$ in this example). The integrals can be evaluated analytically as

$$
\begin{aligned}
& \int_{0}^{L} \int_{0}^{L} \frac{\mathrm{J}_{0}\left(k_{r}\left|s_{2}-s_{1}\right|\right)}{M_{k}} d s_{1} d s_{2} \\
& \quad=\frac{1}{M_{k}} \int_{0}^{L}\left[\int_{0}^{s_{2}} \mathrm{~J}_{0}\left(k_{r} s\right) d s+\int_{0}^{L-s_{2}} \mathrm{~J}_{0}\left(k_{r} s\right) d s\right] d s_{2} \\
& =\frac{L^{2}}{M_{k}}{ }_{1} \mathrm{~F}_{2}\left(\frac{1}{2},\left[\begin{array}{ll}
\frac{3}{2} & 2
\end{array}\right], \frac{-k_{r}^{2} L^{2}}{4}\right),
\end{aligned}
$$

where ${ }_{p} \mathrm{~F}_{q}(\mathbf{p}, \mathbf{q}, x)$ denotes the generalized hypergeometric function with vector parameters $\mathbf{p}$ and $\mathbf{q}$ of length $p$ and $q$, respectively, and argument $x$. The first equality has been obtained with the substitution $s=s_{1}+s_{2}$.

The nonparametric solutions are validated against the full parametric solutions in Figs. 2-5. In Figs. 2 and 3, the mean value of the total energy and the driving point mobility, respectively, is plotted together with the $95 \%$ confidence interval for the whole frequency range. For a specific frequency value, the empirical cumulative distribution function $(\mathrm{CDF})$ is plotted in Fig. 4. An excellent agreement is 

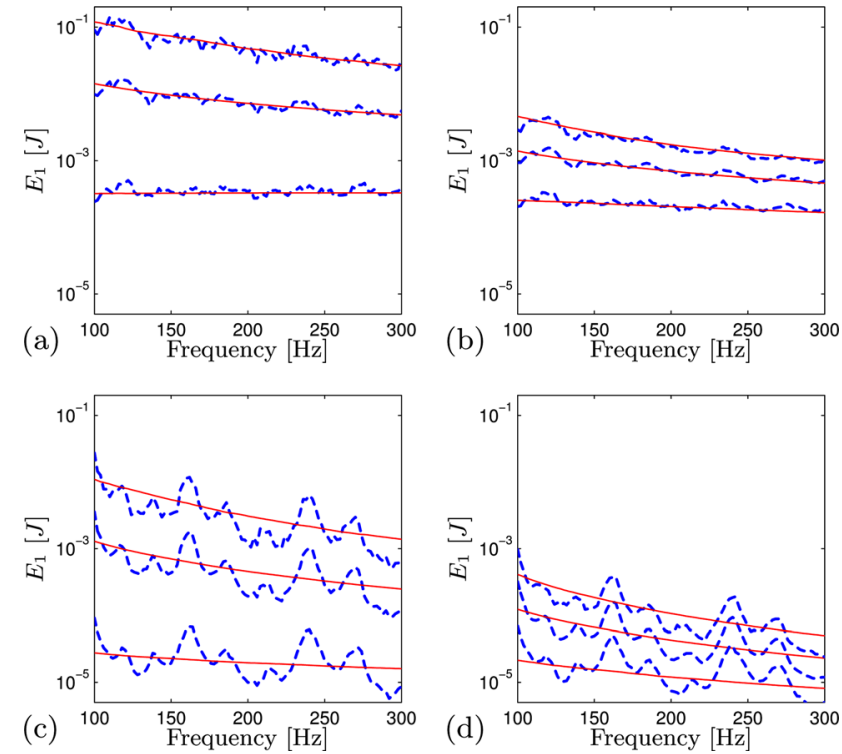

FIG. 2. (Color online) Simply supported plate, total energy $E_{1}$ : mean and 95\% confidence interval for $(a, b)$ point loading and (c,d) line loading, and (a,c) $\eta=0.001$ and (b,d) $\eta=0.01$. Dashed lines: parametric solutions, solid lines: nonparametric solutions.

observed for all loading and damping combinations. This illustrates that the validity of the nonparametric GOE-based approach is not confined to high modal overlap; indeed, for the low damping case $(\eta=0.001)$, the modal overlap $m_{k}$ varies between 0.1 (at $100 \mathrm{~Hz}$ ) and 0.3 (at $300 \mathrm{~Hz}$ ).

The corresponding probability distribution functions, which have been estimated numerically using a kernel density smoother, are plotted in Fig. 5. The good agreement between the parametric PDF and the nonparametric PDF is expected since a similar observation has been made for the CDFs. In the figure these PDFs are compared with a lognormal and chi-squared PDF, which are computed by equating their mean and variance to those of the full parametric simulation. At low damping, neither the lognormal nor the chisquared PDF result in a good fit. In the normal damping case, however, the chi-squared fit is better and the lognormal fit is very good. These observations are in full agreement with the theoretical derivation of the lognormal PDF, ${ }^{7}$ which is only valid at moderate to high modal overlap and for certain loading cases, and that of the chi-squared PDF, ${ }^{8}$ which is only valid at high modal overlap. For low modal overlap,
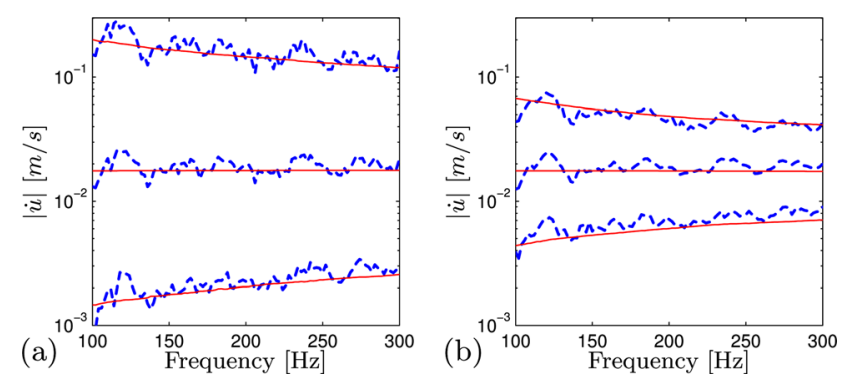

FIG. 3. (Color online) Point loaded simply supported plate, driving point mobility: mean and 95\% confidence interval for (a) $\eta=0.001$ and (b) $\eta=0.01$. Dashed lines: parametric solutions, solid lines: nonparametric solutions.
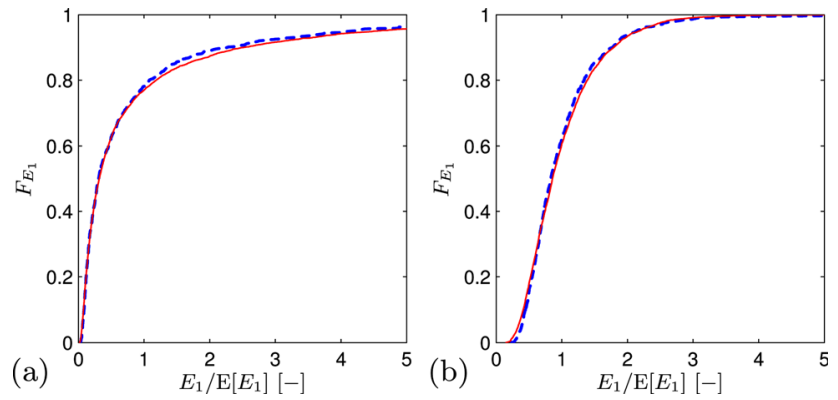

FIG. 4. (Color online) Line loaded simply supported plate, total energy $E_{1}$ : normalized CDF at $200 \mathrm{~Hz}$ for (a) $\eta=0.001$ and (b) $\eta=0.01$. Dashed lines: parametric solutions, solid lines: nonparametric solutions. Results of similar quality are obtained for point loading.

only the nonparametric GOE-based model is capable of capturing the plate's behavior with good accuracy.

\section{B. Oscillator attached to a thin simply supported plate}

Here the same random plate as in the previous section is considered, but now a deterministic oscillator is coupled to the plate at the location of the unit point load [Fig. 1(b)]. The oscillator has a mass of $m=2 \mathrm{~kg}$ and an undamped resonance frequency of $f_{r}=200 \mathrm{~Hz}$. The damping loss factor of the plate and the oscillator is the same. The built-up model of this structure is constructed with the master system approach. The master system contains only a single DOF: the displacement at the connection point. The dynamic stiffness matrix of the master system is therefore a scalar:

$$
\mathbf{D}_{d}=m\left[-\omega^{2}+\left(2 \pi f_{r}\right)^{2}(1+i \eta)\right]
$$

After computing realizations of the nonparametric random subsystem's dynamic stiffness matrix $\mathbf{D}_{1}$, which is of course also a scalar, the corresponding realizations of the master system response are obtained from Eq. (29).

The nonparametric solutions are compared with the full parametric solutions in Figs. 6 and 7. The mean values of the total plate energy $E_{1}$ and oscillator squared velocity amplitude $|\dot{u}|^{2}$ are plotted together with the $95 \%$ confidence interval for the whole frequency range. The cumulative distribution function is also plotted at the undamped resonance frequency of the oscillator. An excellent agreement is observed for both damping cases. Note the particular shape
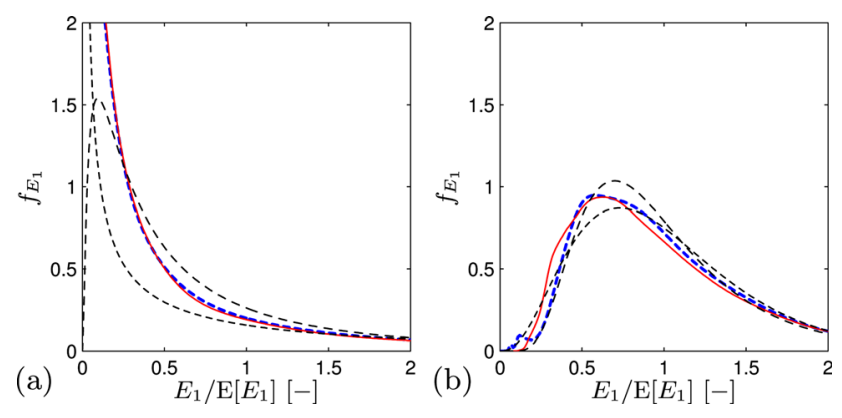

FIG. 5. (Color online) Point loaded simply supported plate, total energy $E_{1}$ : normalized PDF at $200 \mathrm{~Hz}$ for (a) $\eta=0.001$ and (b) $\eta=0.01$. Thick dashed lines: parametric solutions, solid lines: nonparametric solutions, dotted lines: lognormal PDF, dashed lines: chi-squared PDF. 

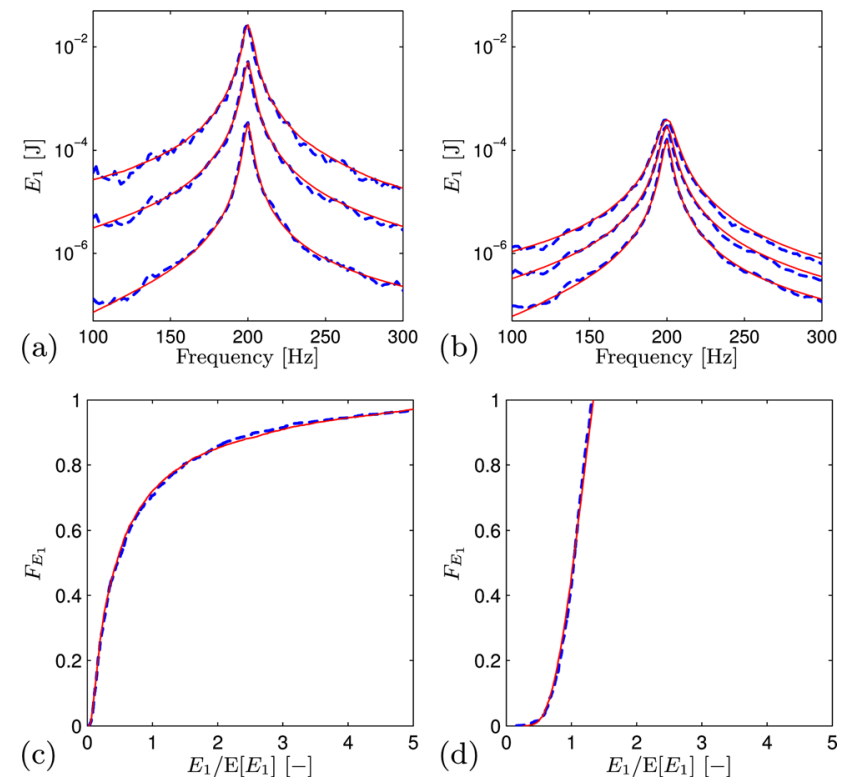

FIG. 6. (Color online) Oscillator attached to a thin plate, total energy $E_{1}$ of the plate: mean and $95 \%$ confidence interval for (a) $\eta=0.001$ and (b) $\eta=0.01$, and normalized $\mathrm{CDF}$ at $200 \mathrm{~Hz}$ for (c) $\eta=0.001$ and (d) $\eta=0.01$. Dashed lines: parametric solutions, solid lines: nonparametric solutions.

of the energy CDF for the normal damping case $(\eta=0.01)$ : there is a sharp cut-off at the higher end of the distribution at around $3.95 \times 10^{-4} \mathrm{~J}$. Neither a lognormal nor a chi-squared distribution would yield a good fit here, as was observed in an earlier study of this particular system. ${ }^{8}$ This cut-off behavior is only observed at resonance and it is therefore attributed to the interaction between the oscillator and the plate at this frequency.

\section{Two plates coupled by a spring}

Next we consider a plate assembly consisting of two flexible, high modal density simply supported plates with random mass distribution [Fig. 1(c)]. The dimensions of the first and second randomized plate are $1.9 \mathrm{~m} \times 2.1 \mathrm{~m} \times$ $1.25 \mathrm{~mm}$ and $2.625 \mathrm{~m} \times 2.375 \mathrm{~m} \times 1.67 \mathrm{~mm}$, respectively. The statistical overlap varies between 3.9 (at $100 \mathrm{~Hz}$ ) and 11.7 (at $300 \mathrm{~Hz}$ ) for the first plate and between 4.6 (at $100 \mathrm{~Hz}$ ) and 13.7 (at $300 \mathrm{~Hz}$ ) for the second plate, so the GOE conditions are fulfilled. All plates have the same
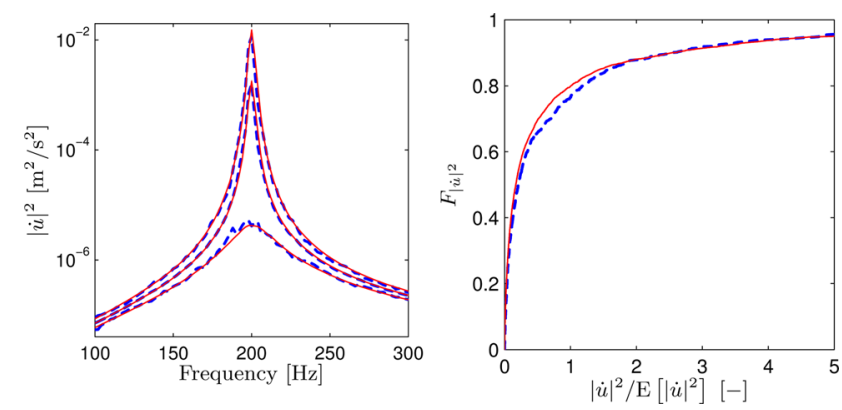

FIG. 7. (Color online) Oscillator attached to a thin plate, squared amplitude $|\dot{u}|^{2}$ of the oscillator, $\eta=0.001$ : (a) mean and 95\% confidence interval and (b) normalized CDF at $200 \mathrm{~Hz}$. Dashed lines: parametric solutions, solid lines: nonparametric solutions. damping; again two values are considered, $\eta=0.001$ and $\eta=0.01$. The randomized plates are connected by a spring with a stiffness of $17 \mathrm{kN} / \mathrm{mm}$. The first plate is connected to the spring at the coordinates $(0.964,1.262)$, which are in meters relative to one corner of the first plate, and the second at $(1.559,0.959)$, which are relative to the corner of the second plate.

In the nonparametric stochastic model, the spring ends together with the loading DOF are taken as the master system DOFs. For the low damping case $(\eta=0.001)$, the nonparametric solutions are compared with the full parametric solutions in Fig. 8, where the mean values of the total plate energies $E_{1}$ and $E_{2}$ are plotted together with the $95 \%$ confidence interval for the whole frequency range. The cumulative distribution functions at a fixed frequency value are also plotted. An excellent agreement is observed. Similar results are obtained for $\eta=0.01$ but they are not reproduced here.

In Fig. 9, an additional comparison is made with statistical energy analysis predictions. The SEA results are computed with the hybrid formulation of Langley and coworkers for computing the mean ${ }^{10}$ and variance ${ }^{11}$ of the response. Within this formulation, the master system consists of the spring connecting both plates, and the random subsystems are the plates themselves. The SEA results differ slightly from the parametric and nonparametric solutions, especially for the low damping case $(\eta=0.001)$. They underestimate the mean and variance of the total energy of the source plate and they overestimate the mean and variance of the total energy of the receiving plate. The differences can be explained by noting that in SEA, the subsystems are assumed to be weakly coupled why no such assumption is made in the nonparametric stochastic method presented in this paper. Finnveden's Gamma criterion ${ }^{17}$ provides a measure for assessing the coupling strength. When $\gamma<1$, the
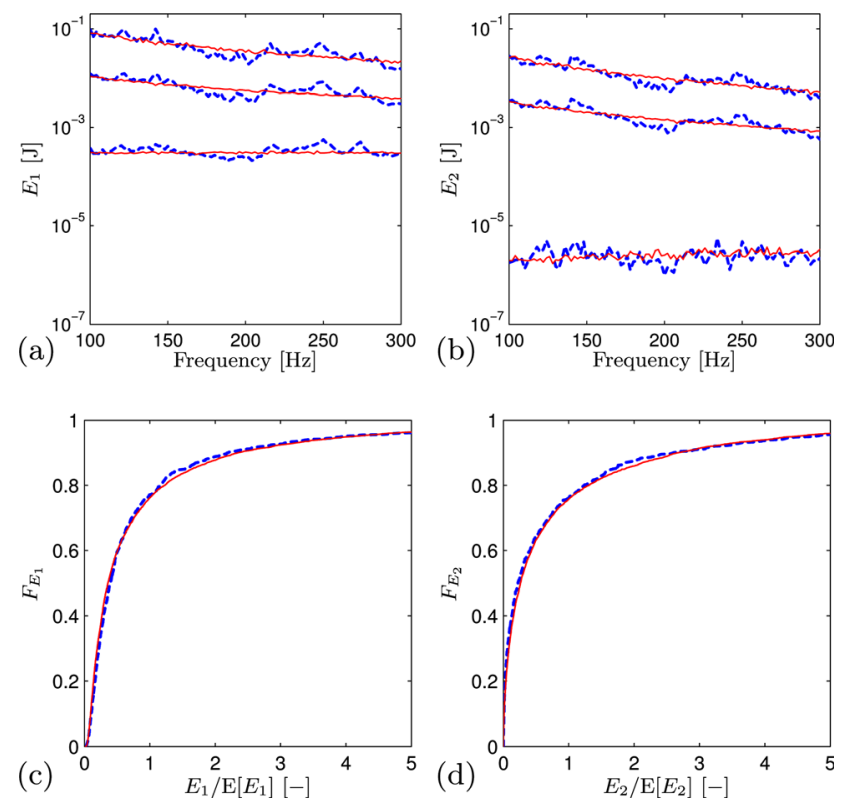

FIG. 8. (Color online) Two-plate assembly, total energies $E_{1}$ and $E_{2}$ of the random plates, $\eta=0.001$ : (a,b) mean and 95\% confidence interval and (c,d) normalized CDF at $200 \mathrm{~Hz}$. Dashed lines: parametric results, solid lines: nonparametric solutions. 

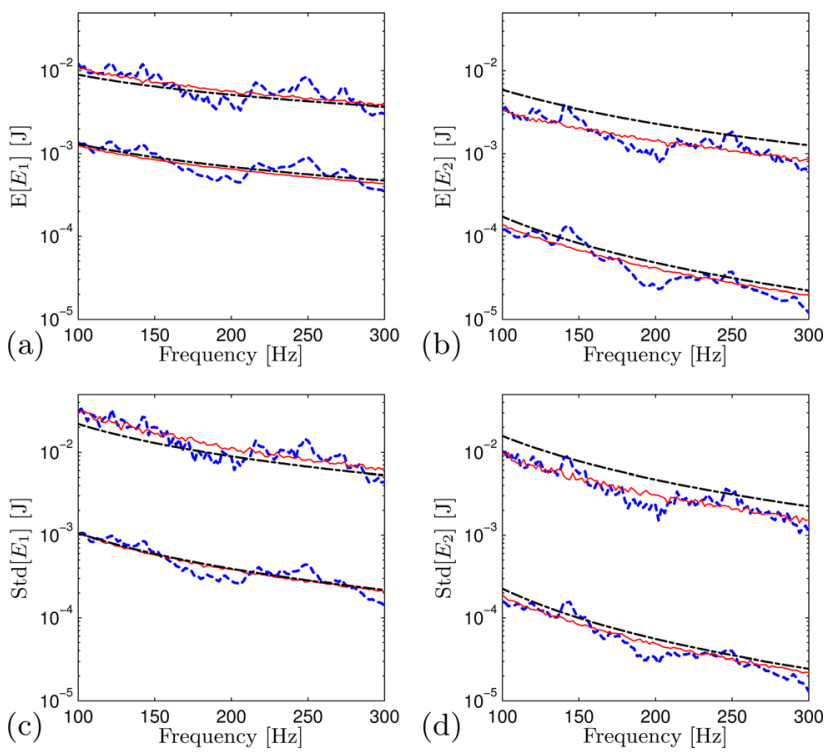

FIG. 9. (Color online) Two plates coupled by a spring, total energies (a,c) $E_{1}$ and $(\mathrm{b}, \mathrm{d}) E_{2}$ : $(\mathrm{a}, \mathrm{b})$ mean values and $(\mathrm{c}, \mathrm{d})$ standard deviations. Upper curves: $\eta=0.001$, lower curves: $\eta=0.01$. Dashed lines: parametric results, solid lines: nonparametric solutions, dash-dotted lines: SEA predictions.

coupling is weak, while the coupling is strong when $\gamma>1$. For the specific case of two plates coupled by springs, $\gamma$ grows when (i) the spring stiffness increases, (ii) the number of springs increases, (iii) the plate damping decreases, (iv) the plate stiffness or mass increases, and (v) the frequency decreases. For the plate assembly considered here, $\gamma \approx$ $3 \times 10^{5}$ at $200 \mathrm{~Hz}$ when $\eta=0.001$ while $\gamma \approx 3 \times 10^{3}$ at $200 \mathrm{~Hz}$ when $\eta=0.01$.

\section{Three-plate assembly}

Finally, we consider a plate assembly where a low modal density, simply supported master plate is connected to two flexible, high modal density simply supported plates with random mass distribution [Fig. 1(d)]. The dimensions of the master plate are $1.05 \mathrm{~m} \times 0.95 \mathrm{~m} \times 5 \mathrm{~mm}$, those of the first and second randomized plate are $1.9 \mathrm{~m} \times 2.1 \mathrm{~m}$ $\times 1.25 \mathrm{~mm}$ and $2.625 \mathrm{~m} \times 2.375 \mathrm{~m} \times 1.67 \mathrm{~mm}$, respectively. The material properties are the same as before. All plates have the same damping loss factor; again two values are considered, $\eta=0.001$ and $\eta=0.01$. The randomized plates are rigidly connected to the master plate, the first one at the coordinates $(0.464,0.762),(0.832,0.525)$, and $(0.161,0.268)$, which are in meters relative to one corner of the master plate, and the second one at $(0.809,0.209),(0.402,0.181)$, and $(0.764,0.723)$. A unit harmonic force is applied at the first random plate.

The nonparametric stochastic model for this structure is constructed with the master systems approach, where the force connection point and the 32 deterministic free bending modes of the master plate that have a natural frequency below $600 \mathrm{~Hz}$ are taken as master system DOFs. The nonparametric solutions are compared with the full parametric solutions in Figs. 10 and 11, where the probability distribution of the random plate energies and the master plate response at location $(x, y)=(0.255,0.142)$ are plotted. A very good agreement is obtained in all cases. It can be seen
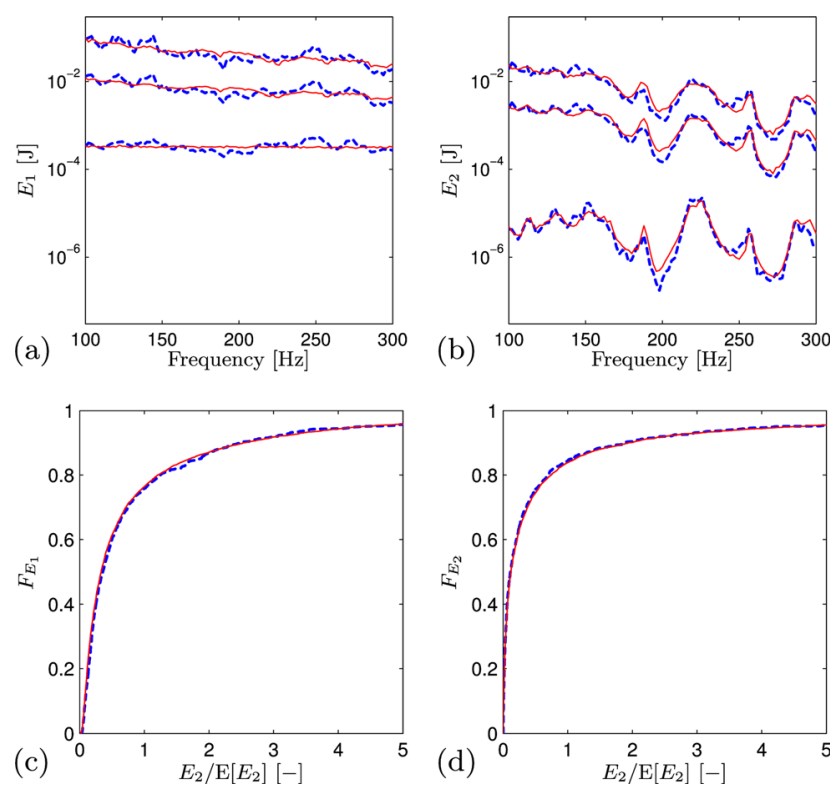

FIG. 10. (Color online) Three-plate assembly, loading on random plate 1, $\eta=0.001$, total energies $E_{1}$ and $E_{2}$ of the random plates: (a,b) mean and $95 \%$ confidence interval and (c,d) normalized CDF at $200 \mathrm{~Hz}$. Dashed lines: parametric results, solid lines: nonparametric solutions. Results of similar quality are obtained for $\eta=0.01$.

in Fig. 10 that the dynamic behavior of both subsystems is qualitatively different. The behavior of random plate 1 , which is directly loaded by a known external force, is not much influenced by the presence of the master plate, and its response is qualitatively similar to that of the detached plate of Sec. IV A. Random plate 2 is loaded through the master plate, and the influence of the individual modes of the master system is clearly visible.

In a previous study of this same example structure, ${ }^{8}$ it was found that the subsystem energies neither follow a
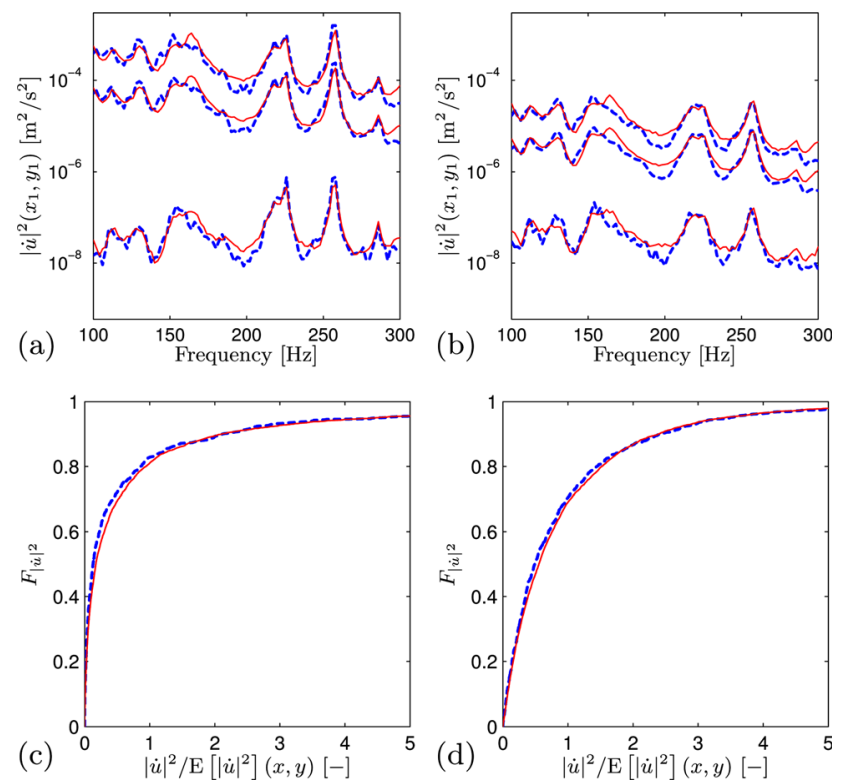

FIG. 11. (Color online) Three-plate assembly, loading on random plate 1, master plate response: mean and $95 \%$ confidence interval for (a) $\eta=0.001$ and (b) $\eta=0.01$, and normalized CDF at $200 \mathrm{~Hz}$ for (c) $\eta=0.001$ and (d) $\eta=0.01$. Dashed lines: parametric results, solid lines: nonparametric solutions. 
chi-squared nor a lognormal distribution, except for the case $\eta=0.01$ and $f>200 \mathrm{~Hz}$, where the chi-squared distribution yields a good approximation. For the master system, an exponential distribution yielded a good approximation at $\eta=0.01$ but not at $\eta=0.001$. With the nonparametric GOE-based approach, a good prediction of the response probability distribution is found in all cases. Results of similar quality (not reproduced here) are obtained when the loading acts on the master system instead of on a subsystem.

\section{CONCLUSIONS}

A nonparametric probabilistic method has been presented for the mid- and high-frequency analysis of built-up structures. The method is robust in the sense that it yields the full probability distribution of the response due to small spatial variations in geometry, material properties, or boundary conditions. It is also computationally efficient because the random subsystems are modeled with the interface DOFs only, and because the natural frequencies and mode shapes of these subsystems are directly drawn from universal probability distributions. The method has a wider range of applicability than statistical energy analysis and related approaches since the full response probability can be accurately predicted for arbitrary loading and arbitrary modal overlap, and since no additional assumptions are needed when coupling a random subsystem to other subsystems - in particular, strong coupling is allowed. Other advantages, which will be exploited in further studies, are that additional parametric uncertainty can be accounted for without increasing the computation cost, that phase information is available so that response signals may be reconstructed in the time domain, and that the computation of band-averaged response statistics is straightforward.

\section{ACKNOWLEDGMENTS}

This research was funded by the Research Foundation-Flanders (FWO), Belgium, under a Postdoctoral Research Fellowship and a Long-stay Travel Grant provided to the first author.

${ }^{1}$ M. S. Kompella and R. J. Bernhard, "Variation of structural-acoustic characteristics of automotive vehicles," Noise Control Eng. 44, 93-99 (1996).

${ }^{2}$ A. Deraemaeker, I. Babuška, and P. Bouillard, "Dispersion and pollution of the FEM solution for the Helmholtz equation in one, two and three dimensions," Int. J. Numer. Methods Eng. 46, 471-499 (1999).

${ }^{3}$ R. Ghanem and P. Spanos, Stochastic Finite Elements: A Spectral Approach, 2nd ed. (Dover, New York, 2003), pp. 1-222.

${ }^{4} \mathrm{E}$. Rosseel and S. Vandewalle, "Iterative solvers for the stochastic finite element method," SIAM J. Sci. Comput. 32, 372-397 (2010).

${ }^{5}$ R. Lyon and R. DeJong, Theory and Application of Statistical Energy Analysis, 2nd ed. (Butterworth-Heinemann, Newton, MA, 1995), pp. 1-277.

${ }^{6} \mathrm{R}$. Langley and V. Cotoni, "Response variance prediction in the statistical energy analysis of built-up systems," J. Acoust. Soc. Am. 115, 706-718 (2004).

${ }^{7}$ R. Langley, J. Legault, J. Woodhouse, and E. Reynders, "On the applicability of the lognormal distribution in random dynamical systems," J. Sound Vib. 332, 3289-3302 (2013).

${ }^{8}$ E. Reynders and R. Langley, "Response probability distribution of builtup vibro-acoustic systems," J. Acoust. Soc. Am. 131, 1138-1149 (2012).

${ }^{9} \mathrm{R}$. Langley, "On the diffuse field reciprocity relationship and vibrational energy variance in a random subsystem at high frequencies," J. Acoust. Soc. Am. 121, 913-921 (2007).

${ }^{10} \mathrm{P}$. Shorter and R. Langley, "Vibro-acoustic analysis of complex systems," J. Sound Vib. 288, 669-699 (2005).
${ }^{11} \mathrm{R}$. Langley and V. Cotoni, "Response variance prediction for uncertain vibro-acoustic systems using a hybrid deterministic-statistical method," J. Acoust. Soc. Am. 122, 3445-3463 (2007).

${ }^{12}$ J. A. Cordioli and V. Cotoni, "Review of some industrial applications of the hybrid FE-SEA method to vibro-acoustic prediction and analysis," in Proceedings of NOVEM2009 (Oxford, UK, 2009), pp. 821-832.

${ }^{13} \mathrm{~J}$. Woodhouse, "An approach to the theoretical background of statistical energy analysis applied to structural vibration," J. Acoust. Soc. Am. 69, 1695-1709 (1981).

${ }^{14} \mathrm{~S}$. Finnveden, "Ensemble averaged vibration energy flows in a three-element structure," J. Sound Vib. 187, 495-529 (1995).

${ }^{15} \mathrm{~B}$. Mace, "Statistical energy analysis, energy distribution models and system modes," J. Sound Vib. 264, 391-409 (2003).

${ }^{16} \mathrm{~B}$. Mace, "Statistical energy analysis: coupling loss factors, indirect coupling and system modes," J. Sound Vib. 279, 141-170 (2005).

${ }^{17}$ S. Finnveden, "A quantitative criterion validating coupling power proportionality in statistical energy analysis," J. Sound Vib. 330, 87-109 (2011).

${ }^{18}$ R. Weaver, "Spectral statistics in elastodynamics," J. Acoust. Soc. Am. 85, 1005-1013 (1989).

${ }^{19}$ P. Bertelsen, C. Ellegaard, and E. Hugues, "Distribution of eigenfrequencies for vibrating plates," Eur. Phys. J. B 15, 87-96 (2000).

${ }^{20}$ C. Ellegaard, K. Schaadt, and P. Bertelsen, "Acoustic chaos," Phys. Scr. T90, 223-230 (2001).

${ }^{21}$ O. Bohigas, M. Giannoni, and C. Schmit, "Spectral properties of the Laplacian and random matrix theories," J. Phys. Lett. Paris 45, 1015-1022 (1984).

${ }^{22}$ R. Langley and A. Brown, "The ensemble statistics of the energy of a random system subjected to harmonic excitation," J. Sound Vib. 275, 823-846 (2004).

${ }^{23}$ M. Mehta, Random Matrices, 3rd ed. (Elsevier, San Diego, CA, 2004), pp. $1-688$.

${ }^{24} \mathrm{E}$. Wigner, "On the statistical distribution of the widths and spacings of nuclear resonance levels," Math. Proc. Cambridge 47, 790-798 (1951).

${ }^{25}$ E. Wigner, "Characteristic vectors of bordered matrices with infinite dimensions," Ann. Math. 62, 548-564 (1955).

${ }^{26} \mathrm{~F}$. Dyson, "Statistical theory of the energy levels of complex systems. I-III," J. Math. Phys. 3, 140-175 (1962).

${ }^{27} \mathrm{R}$. Langley, "Universal eigenvalue statistics and vibration response prediction," in IUTAM Symposium on the Vibration Analysis of Structures with Uncertainties, edited by A. Belyaev and R. Langley (Springer, St. Petersburg, Russia, 2009), pp. 115-128.

${ }^{28}$ R. Cook, R. Waterhouse, R. Berendt, S. Edelman, and M. Thompson, Jr., "Measurement of correlation coefficients in reverberant sound fields," J. Acoust. Soc. Am. 27, 1072-1077 (1955).

${ }^{29}$ M. V. Berry, "Regular and irregular semiclassical wavefunctions," J. Phys. A: Math. General 10, 2083-2091 (1977).

${ }^{30} \mathrm{C}$. Soize, "A nonparametric model of random uncertainties for reduced matrix models in structural dynamics," Probabilist. Eng. Mech. 15, 277-294 (2000).

${ }^{31} \mathrm{C}$. Soize, "Random matrix theory for modeling uncertainties in computational mechanics," Comput. Methods Appl. Mech. Eng. 194, 1333-1366 (2005).

${ }^{32} \mathrm{M}$. Mignolet and C. Soize, "Nonparametric stochastic modeling of linear systems with prescribed variance of several natural frequencies," Probabilist. Eng. Mech. 23, 267-278 (2008).

${ }^{33}$ L. Cremer, M. Heckl, and B. Petersson, Structure-Borne Sound: Structural Vibrations and Sound Radiation at Audio Frequencies, 3rd ed. (Springer, Berlin, 2005), Sec. 5.7.3, pp. 298-306.

${ }^{34}$ C. Manohar and A. Keane, "Statistics of energy flows in spring-coupled onedimensional subsystems," Philos. Trans. R. Soc., A 346, 525-542 (1994).

${ }^{35}$ R. Lyon, "Statistical analysis of power injection and response in structures and rooms," J. Acoust. Soc. Am. 45, 545-565 (1969).

${ }^{36}$ M. Wright and R. Weaver, eds., New Directions in Linear Acoustics and Vibration: Quantum Chaos, Random Matrix Theory and Complexity (Cambridge University Press, Cambridge, UK, 2010), pp. 42-58.

${ }^{37} \mathrm{C}$. Soize, "Random matrix theory and non-parametric model of random uncertainties in vibration analysis," J. Sound Vib. 263, 893-916 (2003).

${ }^{38}$ A. Thite and B. Mace, "Robust estimation of coupling loss factors from finite element analysis," J. Sound Vib. 303, 814-831 (2007).

${ }^{39}$ E. Reynders, J. Legault, and R. S. Langley, "On the response distribution of nonparametric probabilistic models for mid- and high-frequency analysis," in Proceedings of ISMA2012-USD 2012, edited by P. Sas, D. Moens, and S. Jonckheere (Leuven, Belgium, 2012), pp. 4855-4870. 
${ }^{40}$ W. Choi, R. S. Langley, and J. Woodhouse, "Boundary effects on the vibration statistics of a random plate," J. Sound Vib. 332, 850-866 (2013).

${ }^{41}$ M. Eiermann, O. Ernst, and E. Ullmann, "Computational aspects of the stochastic finite element method," Comput. Visualization Sci. 10, 3-15 (2007).
${ }^{42}$ The distribution is not necessarily circular complex-meaning that the real and imaginary parts of $f_{k, r}$ do not necessarily have the same variance-since for instance $f(\mathbf{x})$ can be purely real or purely imaginary.

${ }^{43}$ L. Meirovitch Elements of Vibration Analysis (McGraw-Hill, New York, 1975), Chap.7, pp. 266-299. 